\title{
Decoupling Representations and the Chain of Arguments
}

\author{
CRISTIÁn SANTIBÁÑez \\ Department of Language Sciences \\ Universidad Católica de la Santísima de Concepción \\ Alonso de Ribera 2850, Concepción, 4090541 \\ Chile \\ csantibanez@ucsc.cl
}

\begin{abstract}
In this paper, I propose to understand argumentative decouplingthat is, the structural fact of the argumentative chain self-referring to one (or more) of its constituents (reason, data, conclusion, point of view) in subsequent arguments - as part of the way in which cognitive decoupling representation works. In order to support this claim, I make use of part of the discussion developed in cognitive studies and evolutionary theories that describes this phenomenon when explaining intentional communication. By using Toulmin's model, I exemplify how decoupling representation may be seen as part of a chain of arguments in which a second argumentative move is usually oriented to action. I conclude by reflecting on the relationship between this human cognitive capacity and the problem of recursion to hold that these two concepts are not synonymous but stand in a subordinated and complementary relation to each other.
\end{abstract}

Résumé: Dans cet article, je propose de comprendre le découplage argumentatif, c'est-à-dire le fait structurel de la chaîne argumentative consistant à se référer à un (ou plusieurs) de ses constituants (raison, données, conclusion, point de vue) dans des arguments ultérieurs, dans le cadre du fonctionnement de la représentation du découplage cognitif. Pour étayer cette affirmation, j'utilise une partie de la discussion développée dans les études cognitives et les théories évolutionnistes qui décrivent ce phénomène pour expliquer la communication intentionnelle. En utilisant le modèle de Toulmin, je montre comment la représentation du découplage peut être considérée comme faisant partie d'une chaîne d'arguments, dans laquelle un deuxième mouvement argumentatif est généralement orienté vers l'action. Je conclus en réfléchissant à la relation entre cette capacité cognitive humaine et le problème de la récursivité pour affirmer que ces deux concepts ne sont pas synonymes mais se situent dans une relation subordonnée et complémentaire l'un à l'autre.

Keywords: chain of arguments, cognition, decoupling, mind, recursion, Toul$\min$ 


\section{Introduction}

In this paper, I take Sterelny's (2003) idea of decoupling and apply it to the functioning of a chain of argument. I understand decoupling as the cognitive capacity to use the same piece of information (e.g., a belief) with two or more different roles, that is, information that can have different functions depending on the context of the response to a demand from the environment. The goal of this paper is to develop the idea that decoupling has a specific manifestation in social argumentative activity. Specifically applied to the chain of argument, the concept of decoupling is understood as the use of an element (e.g., premise, conclusion, or warrant) in an initial argument with a particular function that adopts a new function in a subsequent argument, or second move in an argumentative chain. This second function can be seen as a result of the speaker's intention to respond to a demand from the audience or, for example, to complete their argumentative goal.

Several authors in different domains have been reflecting on the phenomena of decoupling. Among other disciplines that have discussed this problem, we can find evolution theory (Christensen 2010), cognitive studies (Dickinson and Balleine 2000), philosophy of biology (Godfrey-Smith 1996), and evolutionary psychology (Cosmides and Tooby 2000). Unfortunately, in argumentation theory, there is no explicit approach to this topic. There are, however, indirect ways of addressing this, such as Hamblin's reflection (1970) on what he calls a complex argument. Hamblin points out that a complex argument is composed of multiple simple ones between which there is a kind of structural borrowing. In legal reasoning, to mention another discipline that addresses this topic indirectly, the phenomenon has been termed sorites.

To initially exemplify the nature of decoupling in the construction of an argument, a closer look at sorites helps. Sorites are a series of arguments chained in such a way that the predicate of the first one is the subject of the second one. Then, the predicate of the second argument is the subject of the third. This discursive process goes on until it reaches a point (conclusion). At that point, the proposition of the first subject comes together with the predicate of the last one as in A is B, B is C, C is D, D is E, then A is E. An 
example of this could be: Infanticides are ruthless criminals; ruthless criminals are society's most dangerous enemies; society's enemies must be severely punished; severe punishments must be exemplary; the exemplary punishment par excellence is capital punishment; then the infanticides must be exemplarily punished with the maximum penalty. Thus, initially, decoupling can refer to the inferential process where an element-reason, data, premise, warrant, conclusion, point of view ${ }^{1}$ - holds a specific initial position in an inferential structure; subsequently, the element has a different role in another structure, and then in another one, and it has a different function from the previous structures in which it participated.

Considering that decoupling is manifested in different cognitive capacities, as will be explained in the following sections, how decoupling is part of the argument's dynamic is discussed here. This paper aims to clarify the functioning of decoupling representation in argumentative dynamics by using part of the discussion developed in both cognitive studies and philosophy of biology. In the following section, the notion of decoupling is discussed particularly following Sterelny's (2003) approach from a philosophy of biology point of view; then in section three, the problem of decoupling is further characterized by using some elements from the discussion within developmental studies of cognition in infancy, specifically analyzing it from an ontogenetic angle; in the fourth section, Toulmin's model of argument reconstruction is used to exemplify the phenomenon of decoupling representations; in the final section, I compare the concept of decoupling to the notion of recursion to consider whether decoupling pertains to a broader phenomenon of the mind. This paper is a tentative explanation of an empirical and theoretical challenge.

\footnotetext{
1 Both the terms and the relationships between terms vary according to the theory or conceptual framework. For instance, we have the reason-point of view relationship in the pragma-dialectical theory (van Eemeren and Grootendorst 2004), data-claim in the Toulminian model (Toulmin 1958), and endoxon-dataconclusion in the Lugano school's approach (Rigotti and Greco 2019).
} 


\section{Decoupling: What is it?}

One way to approach decoupling is through Sterelny's (2003) general notion of translucent communication, which captures the context in which decoupling emerges from an evolutionary and cognitive perspective. According to Sterelny's view, it is first essential to understand that our mental capacity has increased as a result of our cognitive history, analogically to other species, to strategically respond to an environment that manifests all kinds of contingencies. Therefore, we should assume significant cognitive plasticity in human behavior.

According to Sterelny (2003), organisms evolved equipped with control systems so that they can survive in highly competitive environments. These control systems are presented as detection systems (e.g., of food sources, of signs of threat, or of possible mates). These control systems can generate, with a certain degree of accuracy, adaptive behavior in transparent environments. In other words, in those transparent environments where the contingencies do not make the signs vary brusquely, the demand is low in terms of information processing. But in translucid environments, that is, where a sign can have more than just one value and the agent is forced to adopt more sophisticated behavior to respond correctly to a contextual challenge, the detection system is not enough, and therefore the evolutionary pressure generates robust tracking mechanisms.

The robust tracking mechanism is a cognitive function prepared to deal with complex environments composed of many variables acting at the same time (in terms of agents, navigating information, expectations, etc.). This requirement is especially necessary when the context is, epistemically speaking, less traceable. ${ }^{2}$ In such a context, the organism creates a connection between one of the environment's tracked aspects and the richness of its behavioral response. For example, an agent can receive what seems to be a warning signal indicating that a threat is getting closer, such as a predator in the vicinity. The phrase seems to be a warning is used here because when it comes to scanning or tracking a problem, the

\footnotetext{
2 The phrase "less traceable" in signal communication theory can be seen as synonymous to the concept of vagueness in pragmatics.
} 
agent might evaluate the elements in the environment as something different from what they are. Examples of this could be the presence of sounds that hinder the clarity of a signal, the use of previous information to evaluate the impossibility of a predator being around, or knowing beforehand that other agents emit specific signals in that environment that are known for causing unnecessary alarm. All the possibilities that this agent could consider when deciding upon a course of action are a manifestation of the richness of its behavioral response. The agent is equipped with this behavioral richness partly from heritage, partly from accumulation and experience, and partly from trial and error. This richness allows them to respond in a variety of ways depending on how they contemplate the set of variables. If the agent errs in their judgment, they may pay dearly for the error. But if they do not err, the benefits may increase. Hence, high cognitive flexibility is a more powerful strategy than a mechanism that highly discriminates between two values (yes/no, existence/absence). The weakness of high cognitive flexibility can be an extreme sensitivity to continually revising alternative responses to the environment. While the first cognitive strategy (high flexibility) allows the agent to have multivariable responses to the environment, the second one (highly discriminative) allows unnecessary confusion to be avoided. The problem involved in the first cognitive strategy is a risk to integrity due to the interpretation of many factors at the same time. As for the second strategy, it endangers integrity when it results in overestimating only one sign that could, at the same time, be the strategy used by another agent for their benefit (e.g., free riders).

As Sterelny (2003) has pointed out, to adapt more successfully, an organism is expected to be capable of creating representations of the variables that are at stake in actional and communicative contexts. Intentional systems are the ones capable of doing so. When sophisticated intentional systems, like humans, use robust tracking mechanisms, they guide their actions using decoupled representations: records of the environment that are relevant for several possible actions but, functionally speaking, are not specific to any of them. 
An example that can clarify the role of decoupling representations in reasoning processes ending in a behavioral decision is mothers 'reading' their baby's cry. ${ }^{3}$ The baby's cry is a piece of information similar to a warning sign in the animal kingdom, but because of the mother's richness of behavioral responses construed by experience, trial and error, or memory, she evaluates other environmental clues to decide whether the cries correspond to pain, hunger, or tiredness, among other possibilities. The representation baby crying is a record of an interaction between intentional human agents not functionally determined by any specific course of action; it is detached from an automatic response. This example also helps show that decoupled representations between human agents are extremely important given that we decide upon courses of action by considering expectations (our own and those of others) and potential deceptive behaviors and by using mind reading to accommodate our decisions regarding possible future (desirable) outcomes. ${ }^{4}$

This cognitive definition of decoupling representations somewhat resembles a lexicographic definition. At a lexicographic level, decoupling refers to the separation of parts belonging to a unit that can functionally detach itself from its constituents without any of them, in principle, being damaged afterward. They could, on the contrary, end up obtaining more autonomy. From the cognitive perspective, the notion of decoupling has to do with representations that detach themselves from their precedent uses but inside

\footnotetext{
${ }^{3}$ I am thankful to the critical reviewers who asked for more examples to clarify the basic functioning of decoupled representations.

${ }^{4}$ This is the reason why Sterelny (2003) uses the notion of decoupled representation linked to the intelligent social hypothesis to explain human cognitive behavior. As social creatures, we must balance motivations, expectations, and distribution of power and hierarchy among the members of in and out groups. Sterelny explains this straightforwardly as follows: "Cognitive tracking is neither wholly cue-bound nor rigidly tied to specific behaviors ... More generally, I think the basic thrust of the social intelligence hypothesis is right. The fact that social interaction is strategic, and the fact that there is a feedback loop built into this picture of cognitive evolution, makes it easy to agree both that selection for social intelligence was an important element of primate cognitive evolution, and that social life was an important driver of the evolution of belieflike states in our lineage. Decoupled representations may well have had their origin in social maps" (2003, p. 76).
} 
a brain-mind container that can reintegrate them into their previous uses. For this reason, their autonomy is contextually dependent, functionally goal-oriented, or both at the same time.

Both Sterelny (2003) and his critics (e.g., Christensen 2010) acknowledge that it was Godfrey-Smith (1996) who brought attention to the need to think more thoroughly about how environmental complexity selects flexible behavior. To go deeper into the phenomenon, these authors agree that an organism's flexible response to environmental variation is adaptive when the benefits of detecting the variation and the response towards it exceed the costs. Faced with this, Sterelny describes a succession of increasingly sophisticated forms of sensorimotor control, beginning with detection systems that show specific adaptive responses to signals from the environment. An agent that is only a detector is, consequently, equipped with detection systems (for example, an organism that has refined its sense of smell to detect threats that have a particular smell). As Christensen accurately summarizes, these organisms behave functionally in a compelling manner. Moving upwards in the behavioral complexity scale, the next step, robust tracking, involves tracking the environment's relevant features using several crossover indications (from the environment itself to the experience the organism accumulates). This culminates in a cognitive scale with the disposition of decoupled representations that Sterelny (2003) defines as “...internal states that track aspects of our world, but which do not have the function of controlling particular behaviors" (p. 29).

From an evolutionary perspective, the detection system is phylogenetically more restricted than the robust tracking system, and the latter is restricted regarding decoupled representations. ${ }^{5}$ Species with high adaptability - that is, those that struggle with environmental variability - display the use of decoupled representations. These decoupled representations are notably common in the case of humans who deal with hostile, aggressive environments

\footnotetext{
${ }^{5}$ I will leave out the productive discussion that Christensen (2010) develops in his critique of Sterelny related to ambiguity and synonymy in signals. In a nutshell, ambiguity occurs when multiple environmental conditions map with sensory discrimination and synonymy when a given environmental condition maps with multiple sensory discriminations.
} 
and frequent competition for survival resources. From the human ontogenetic point of view, a simple way to facilitate flexible behavior - through learning, for instance - is to order the production of sensitive behavior employing a type of structure made up of the recent history of responses. Thus, in the case of habituation, the production of responses declines when the stimulus has continuously been presented. The fact that we humans are constantly learning is a shred of clear evidence that we do not live in transparent conditions. As a result, social learning is essential for us (Hoppitt and Laland 2013), and perhaps that is the reason why we operate using decoupled representations.

\section{Decoupling from an ontogenetic point of view}

In the arena of infant cognition studies, decoupling has been analyzed with a slightly different emphasis, although the core idea is maintained. This approach helps to elucidate the developmental dimension of the functioning of decoupled representations. In describing the system of infantile reasoning, Baillargeon et al. (2013) embrace the hypothesis of joint action understanding of false beliefs in children and describe two subsystems. The first one allows children to attribute both motivational states (goals, dispositions) and epistemic states (what the agent knows and does not know) to the agents with whom they are interacting. Subsystem 2 expands subsystem 1, allowing for counterfactual states to be attributed to agents, including false beliefs and pretend beliefs. When an agent offers information about a scene that is incompatible with the information available to the child, system 2 allows the infant to represent those divergences. This decoupled mechanism specifically allows a separate representation of the scene to be created that incorporates the false belief and/or the intended belief of the agent, which enables the infant to predict and interpret the future or potential actions of the agent. Baillargeon proposes this division after evaluating neurophysiological evidence showing the difficulty people with autism have representing false or pretend beliefs in the agents with whom they interact, which points to an inability to uncouple from the direct environment. 
The limitations of human agents on the autism spectrum who function mainly using reasoning sub-system 1, particularly the incapacity to attribute false or pretend beliefs to the individuals with whom they interact, is similar to the limitations of agents equipped only with the control system to scan the signs of the environment, because having a system that discriminates only between two values (yes/no, existent/inexistent, presence/absence, available/non-available, etc.) reduces the alternatives to further evaluate other variables or, more importantly, to consider hidden intentions.

Conceived as the cognitive ability to (1) change perspective, (2) adopt that of the opposite agent, (3) withhold information, and (4) uncouple from the immediate, direct, and transparent environment, the functional spectrum of subsystem 2 is compelling and of fundamental importance, ranging from momentarily incorporating false beliefs to participating in make-believe worlds. Baillargeon attributes a crucial role to this cognitive capacity in the evolution of groups and their members. The origin of subsystem 2 is an evolutionary response to the pressure of maintaining the positivity of the group to which one belongs. Positivity means preventing aggressive confrontations, avoiding pain and uncomfortable emotions, softening uncomfortable situations, or expanding feelings of trust. Baillargeon and her team have carried out various experiments with infants to test this hypothesis of joint action, which has allowed them to understand and explain why we do not care so much about deception at times and why we accept fake things and pretend feelings. The main reason for this is that decoupling from direct experiences and stimulus enables us to put ourselves in another's shoes.

\section{The case in arguments: decoupling dynamics in an argu- mentative chain}

The previous discussion shows that the cognitive capacity of decoupling representations is a pervasive human trait that can be manifested at different levels of cognitive functioning. Argumentative cognitive ability is not an exception. In what follows, I intend to show why this is the case. 
The following example illustrates the dynamic of decoupled representations within an argument. A document produced by a building complex committee and provided to the residents stated:

Our Security Guards' salaries have remained the same for two years, even though the annual CPI has increased $17 \%$ in the last two years. ${ }^{6}$ Because they have urgent needs just like everyone else, we request the authorization of an increase in their monthly payment by $\$ 1,000$ (one thousand pesos). This increase could serve as a backup when demanding more from them in their functions (Rivano 1999, p. 37).

This argumentative text is a piece of practical reasoning in which a request for a specific action is advanced using reasons and rhetorical strategies to justify it, which is something that any analyst would be able to detect quickly. For the present purposes, what is essential here is first, the structural part and then the content of the document. Applying Toulmin's (1958) argument diagram, ${ }^{7}$ the structural reconstruction could be as follows:

\footnotetext{
${ }^{6}$ The CPI (Consumer Price Index) is one of the Chilean economic parameters used to fix salaries. The CPI is the measure or indicator of the fluctuation of prices and the monthly cost of living.

${ }^{7}$ Here I follow the diagram of argument as presented in Toulmin, Rieke, and Janik (1979) - that is, the backing on top, with the warrant vertically below, and the data and conclusion below the warrant diagonally straight out, instead of Toulmin's original model (1958), which had the backing at the bottom, followed by the warrant above, and then the data and conclusion. (Qualifiers and rebuttals, when present, were presented in between data and conclusion).
} 


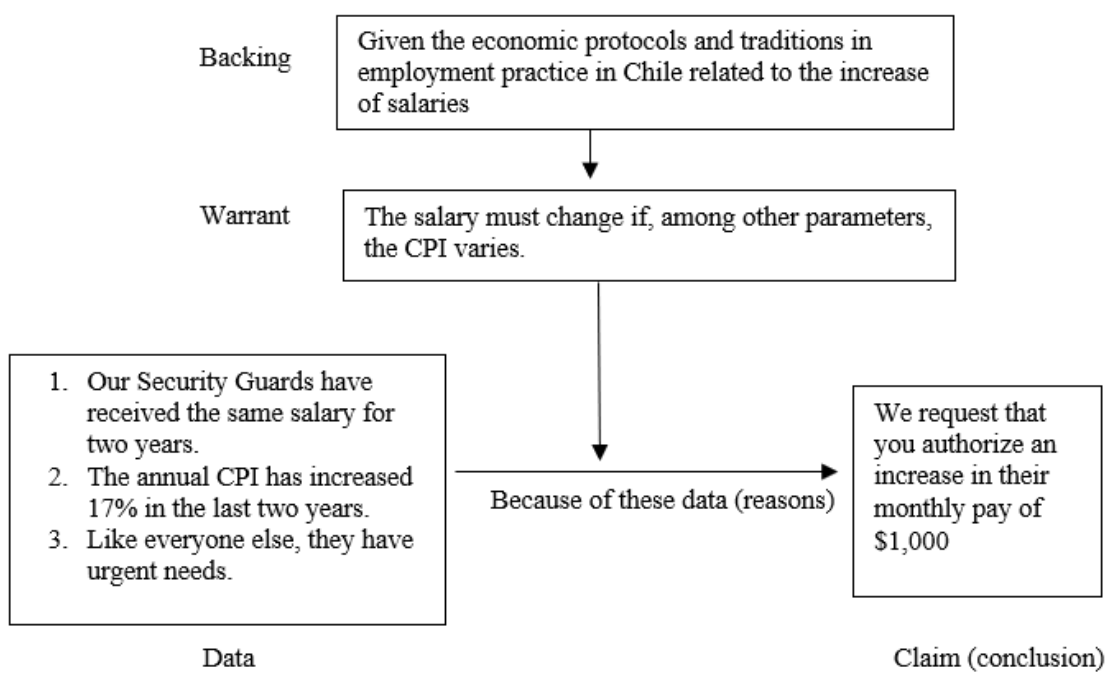

Figure 1.Basic argument

The diagram shows that three facts are being used in the main argument to support the claim. From an argumentative dialogical perspective, this document tries to impose the acceptance of the request on the recipient by appealing to two good reasons: 1 and 2 . Fact 3 is a weak reason since it appeals to emotionscompassion - in a very vague semantic construction (it is difficult to assess what is meant by "Like everyone else, they have urgent needs"). Fact 3 is clearly a strategic move but advanced in a very manipulative way.

As mentioned, what matters here is the structural dynamic of the argument since what is fundamental is implicit in the letter from the committee and unfolds in a reconstruction of the second move contained in the main argument: 


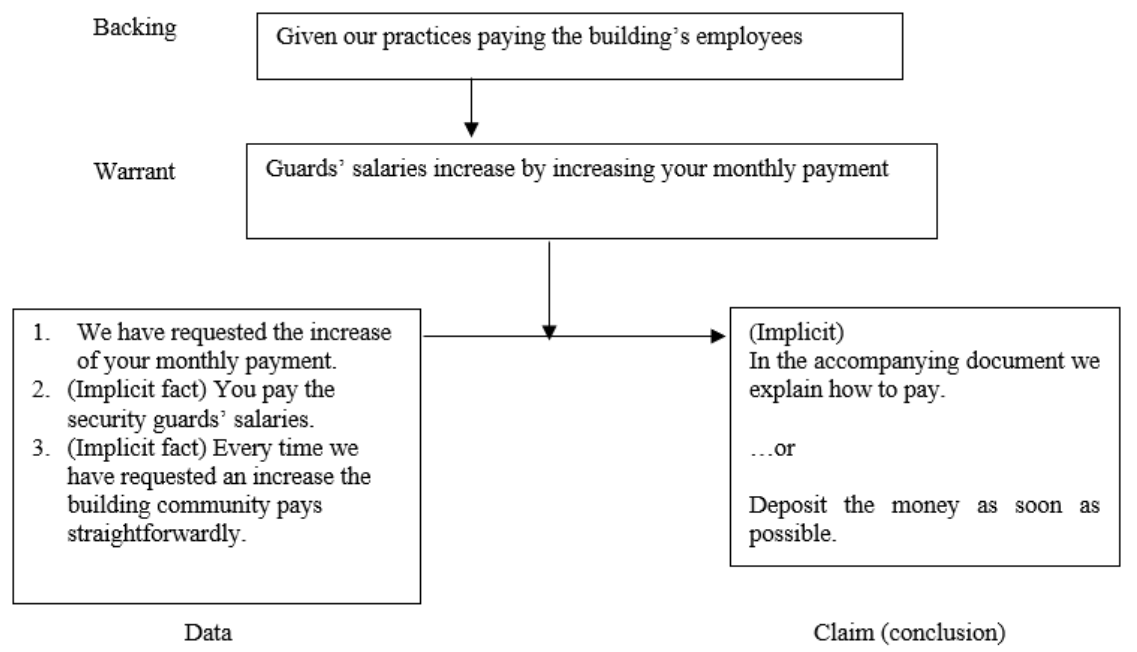

Figure 2. Decoupled argument

In this reconstruction that is oriented to the specific action, the warrant is the conclusion of the main argument. It is the warrant because it becomes a rule of passage in virtue of a collective acceptance of a belief that potentially organizes several actions relative to the domain, or argumentative field, where it is operating. Note that depending on the reconstructions, the conclusion of the main argument may be a data (fact) in the second (actionoriented) argument. From the point of view of the content of the argument in this specific example, the (institutional) speaker (the committee of the building complex) advances the action-oriented argument enthymematically, and many of the reasons are implicit. These implicit reasons must be added to fully grasp the goal of the speaker, ${ }^{8}$ which is, in this case, to effectively ensure the increase of the monthly payment of the residents of the building complex.

It is worth noting that by reconstructing how decoupled representation functions in this way, a particular feature of the dynamic of the argumentative chain is manifested, namely, a transitional

\footnotetext{
${ }^{8}$ Recovering unexpressed premises is one of the natural cognitive automatic activities that any speaker does to understand an argument, which has been labeled theoretically, for example in pragma-dialectics theory, as addition (Van Eemeren 2018). The other three linguistic transformations used to reconstruct arguments in pragma-dialectics are deletion, substitution, and permutation.
} 
component. In the case of the example, first a theoretical (or contemplative) argument is put forward pointing out the state of the affairs, then a practical argument is advanced in the second move that requests a specific action. ${ }^{9}$

This way of framing the argument's transitional dynamic is similar to how Toulmin, Rieke, and Janik (1979) understood the notion of train of argument or chain of reasoning: "An argument, in the sense of a train of reasoning, is the sequence of interlinked claims and reasons that, between them, establish the content and force of the position for which a particular speaker is arguing" ( $p$. 13). Thus, a closer look at an example explaining the notion of a chain of reasoning is also helpful here. Rieke and Sillars (1993), when reflecting on practical reasoning (or decision-making reasoning), use a similar example to the one provided above (Fig. 1 and 2 ). These authors explain the chain of reasoning in the following terms:

Remember, the purpose of a case is to generate adherence to your proposition by the immediately appropriate decision makers. That means the series of claims included in the case must combine to move the decision maker from where they are to where you want to them to be. If you propose to co-workers after a particularly tough job, "Let's order in pizza for the whole crowd", and everyone agrees, your case is made. If, however, your proposition stipulates that the boss pay for the pizza and the boss does not cheer, you need to make a more elaborate case ... Chain of reasoning: You might use a series of claims that moves from ones on which the decision maker is virtually certain to agree (identify a starting point) and move in small steps of adherence to the proposition itself. In the pizza case, it might look like this: Claim one (a starting point): The boss wants workers who are highly productive... Claim two (still virtually a starting point): Happy and satisfied workers are the most productive. Claim three (starting to draw the boss toward the proposition): Workers who are given treats when they do good work are happy and satisfied... Claim four (starting the curve toward the plate): This group has just finished doing a good job. Claim five (this is the kicker): Now is the time for a

\footnotetext{
${ }^{9}$ I prefer to use the notion of transitional instead of, for example, serial, to avoid any confusion with the definition of serial argument proposed by Walton and Gordon (2012).
} 
treat as an investment in higher productivity by this work force (Rieke and Sillars, 1993, p. 72).

Following a Toulminian arrangement, the reconstruction of the main argument in Rieke and Sillars's example might be as follows:

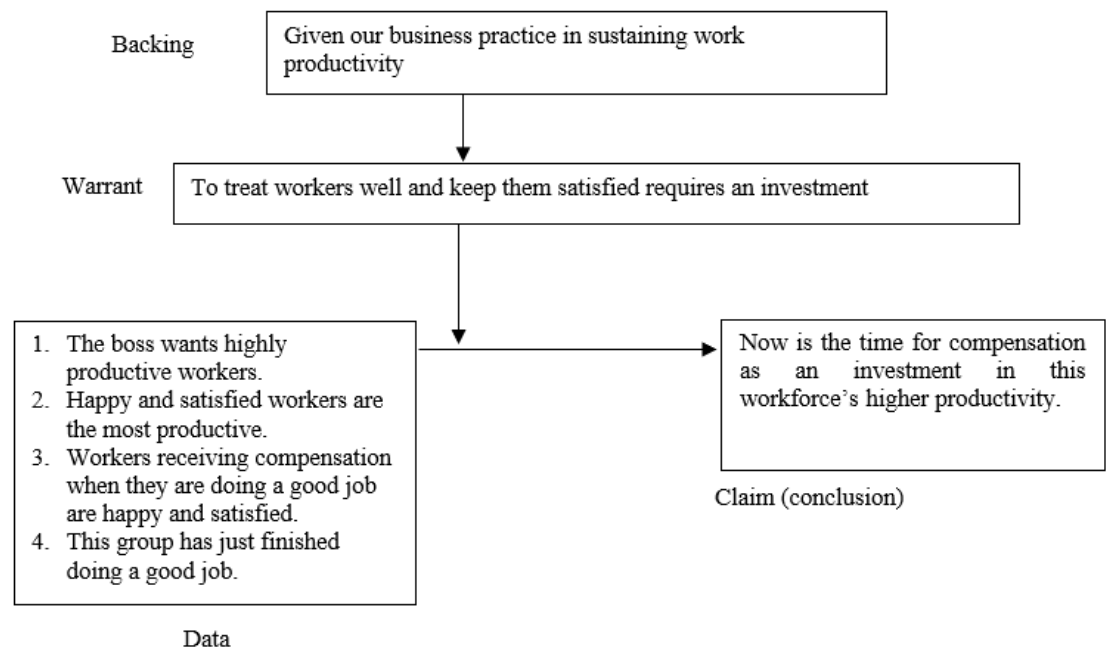

Figure 3. Basic argument

The second move of this chain of reasoning, where some statements are implicit, as the quote from Rieke and Sillars also implies, can be reconstructed as follows similarly to Fig. 2:

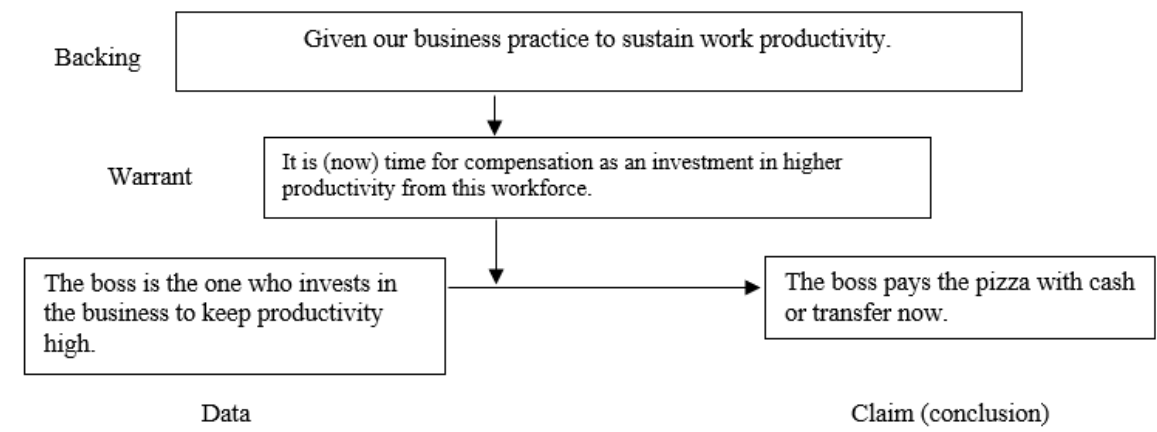

Figure 4. Decoupled argument 
In the warrant in Fig. 4., I have reproduced the content of the conclusion in Fig. 3 almost exactly as it is, but in parenthesis I have annotated the indexical of time (or time adverb) "now." Notice that the time indexical is also recovered in the conclusion of the practical argument moving to the action. The warrant could also be reconstructed in a more rule-like manner, something like: "Every time the workforce does a good job, the investment has to be made immediately." For now, it does not matter whether the warrant has a general force produced by rule-like syntax, if its content is true, or if it is acceptable. The diagram of what I will call from now on the transitional decoupling dynamic of arguments (in virtue of what the examples show) is the result of simplifying and at the same time trying to capture the nature of the argumentative motion and is shown in Fig. 5. One can recall the generative grammar theory to represent recursive syntactic order (Roeper and Speas 2015). 


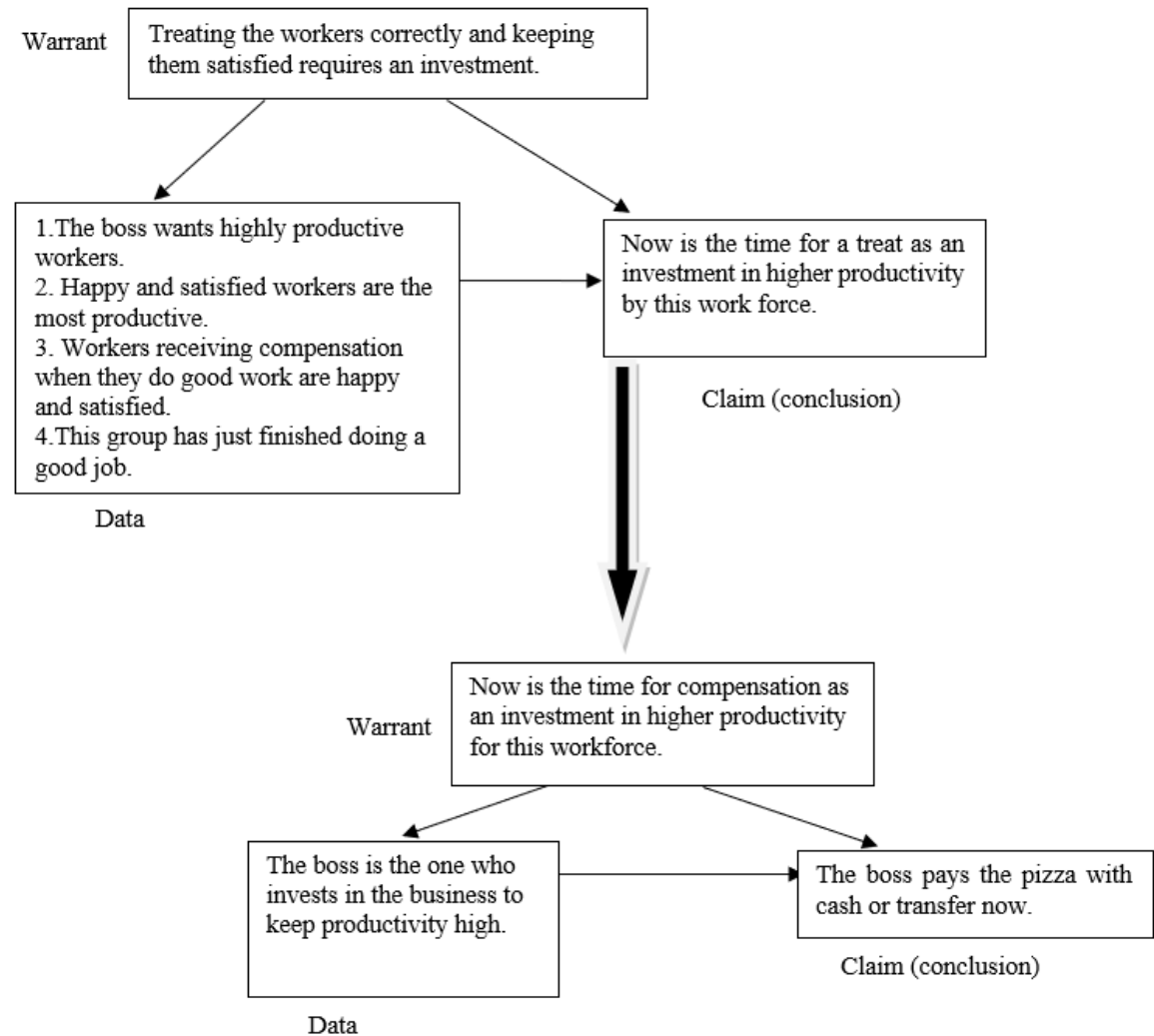

Figure 5. The transitional decoupling dynamic of arguments.

There are some observations to be made regarding Fig. 5. First, the original Toulminian category of backing is left out of the diagram because it does not contain valuable information for understanding the phenomenon of decoupling. Second, to grasp the functioning of the transitional dynamic of the conclusion (of the first or main argument) becoming warrant (in the second or subordinate argument), a different time scale must be in place. Hence, contingent agreements or acceptance allow specific rules to be configured for the second subordinated argument oriented towards an action. Third, the decoupling transitional dynamic ends when the target is reached, but the decoupled representation will be part of the commitment store, a là Hamblin. Fourth, the particular content (conclusion in the main argument, warrant in the sub- 
ordinated one) now has various functions, but it is not specific to any of the steps in the reasoning chain.

Another question would be how long the loop of the argumentative chain can be. The answer, even though it might be obvious, is worth making explicit: it depends on, at least, a fourfold package: (1) the speaker's skills, (2) the contents communicated or under transaction, (3) the audience's reactions, and (4) the goals of the argumentative dialogue in which such an argumentative chain manifests itself (Walton and Krabbe, 1995). This latter idea reveals yet another aspect of the argumentative functioning of decoupled representations: they reproduce and ensure the semiotic commitments a community holds regarding shared assumptions and values (Enfield, 2013). The cultural transmission that the argumentative activity allows when decoupled representations are in use would be as follows: once a collective belief is communicated, we become authorized to use that content as a rule-or as a fact if it does not yet possess collective strength-for the group's immediate or potential future behavior. Henrich (2016) provides an alternative explanation for the ulterior cognitive function of decoupled representations linked to this latter idea. According to him, decoupled representations constitute a strategy for the collective brain and the construction and maintenance of culture. Henrich develops this thesis by mentioning different technological and cultural human achievements to show that during the last 200,000 years, cultural evolution has led to genetic evolution. The larger the size and density of the connections and group relations, the more technological the achievements and solutions, which has an impact on producing individual agents who are better cognitively equipped. Because of this, our generation is better equipped than my mother's. Subsequently, my son's generation is much better equipped than mine. Therefore, decoupled representations are specific to densely connected species, which allows their users to be part of more ecologically differentiated groups. Like two-faced Janus, decoupled representations possess a double-faced flexibility that ensures collective beliefs and allows, when necessary, for variety or dispersion. The argumentative use of these decoupled representations creates an understanding and acceptance of the contents they entail. Cognitively speaking, argumentation is the 
technological tool that allows the learning, apprehension, and communicability of decoupled representations.

Facts, reasons, premises, warrants, backings, points of view, among other terminology, are records of the environment, and they are part of the domain of beliefs fabricated for action, which are relevant to different courses of action. These records are multioriented and non-functional to any scenario or course of action. This cognitive plasticity is what makes us sophisticated intentional structures.

\section{Conclusion: Recursion?}

Recursion is the excluding feature that distinguishes us from the rest of the animal kingdom. This is an emphatic position that is held by Corballis $(2007,2011,2014,2017)$, among others. Chomsky (Berwick and Chomsky, 2016) has always taken a similar stand, though referring exclusively to the linguistic faculty, particularly as a syntactic property. The display of decoupled representations could be considered an expression of recursion as much as a manifestation of the mind's capacity to self-reference, over and over again, its process and contents.

Corballis' explanation of the recursive mind is of the most interest because it converges several dimensions that have already been discussed regarding decoupled representations in general and decoupled representations in the argumentative chain. Corballis functionally describes recursion as follows: "One of the characteristics of recursion, then, is that it can take its own output as the next input, a loop that can be extended indefinitely to create sequences or structures of unbounded length or complexity. In practice, of course, we do not get caught up in infinite loops -life is simply too short for that" (2011, p. 5-6). Corballis (2011, p. 7), following Pinker and Jackendoff (2005), distinguishes between a recursive structure and a recursive process. While the first is the product of a recursive process that does not in itself require being read as recursive (like in the random case of incrusted musical routines), the second one is the manifestation of the incrustation's operations that intentionally possess a system for the generation of meaning. 
Corballis emphasizes the limitation of recursive loops by contingent needs, such as the fact that the agents' planning is organized with and in time. The use of resources (information) from the past for present and future scenarios could have created incrusted structures and processes of (self)reference to maintain the consistency and coherence of the experiences about one's self and the surrounding's phenomena in temporary terms. This capacity is an exceptional evolutionary achievement; no primate, as far as it is known, has been shown to be capable of domesticating this cognitive talent.

An example helps to illustrate the above. Consider an experiment where an actor hides a treat. For any person to be able to grant wishes and express beliefs or even intentions to an actor, they must structure the incrusted sequence that captures what the actor knows about hiding the treat. Daily-life cases like this cause Corballis to hold that the mind, on a very elemental level, is recursive: "[...] in the sense that it involves the insertion of what you believe to be someone else's state of mind into your own" (2011, p. 133). It should be added here that this is exactly the meaning of decoupled representations that Baillargeon et al. (2013) discuss when describing system 2 of the child's reasoning capacity, detailed in section 3 of this paper.

Other philosophers also assume this perspective. Dennett $(1983,1996)$ has a similar view on the matter when explaining the increasing complexity of intentional agents, which leads him to propose the level of intentionality of agents. Dennett categorizes those who can recursively operate as members of the third intentionality level. The author proposes that this level corresponds to an agent who can incrust their intentionality in the other's intentionality. For both authors (Corballis and Dennett), as for Henrich (2016) above, this is the result of an increasing number of interactions and the memories that we have of them, which, in turn, expand the strength and influence of the episodic memory.

The relationship between decoupled representations and recursion becomes apparent using the notion of a loop. Corballis' (2007) paraphrasing of Dunbar's explanation of religious thinking points out that the recursive loop necessary for religious thinking assumes that someone supposes that someone else thinks that they 
believe there are gods who influence our future because they understand our desires; in other words, the recursive loop links mental states to verified/proved intentional states, while the argumentative loop inferentially links information to guide verified or proved intentional states for new purposes (goals, actions). Decoupled representations display cognitive versatility that argumentative practice unfolds.

So, recursion is the general mind structure (Corballis 2011) that allows agents to incrust in their own inferential processes and pieces of information (all kinds of stimuluses) that self-refer to the process itself in loops that progressively permit the agent to understand something or take a course of action. This general process is captured materially and displayed through argumentative competence and the capacity to advance points of view and back them with reasons. In this picture, decoupled representations facilitate the construction of loops in the argumentative chain by providing facts, warrants, and reasons that refer to themselves to strategically build meaningful opinions or states of affairs. Thus, instead of recursion and decoupled representations being the same phenomena, they are in a subordinated and complementary relationship to each other for the benefit of human agency.

\section{References}

Baillargeon, René, Zijing He, Peipei Setoh, Rose Scott, Stephanie Sloane and Daniel Yang. 2013. False-belief understanding and why it matters: The social-acting hypothesis. In Navigating the Social World. What Infants, Children, and Other Species can Teach Us, eds. Mahzarin Banaji and Susan Gelman, 88-95. New York: Oxford University Press.

Berwick, Robert and Noam Chomsky. 2016. Why only Us Language and Evolution. Cambridge: MIT Press.

Christensen, Wayne. 2010. The decoupled representation theory of the evolution of cognition - a critical assessment. Brit. J. Phil. Sci. 61: 361-405.

Corballis, Michael. 2007. Recursion, language, and starlings. Cognitive Science 31: 697-704.

Corballis, Michael. 2011. The Recursive Mind. The Origins of Human Language, Thought, and Civilization. Princeton: Princeton University Press. 
Corballis, Michael. 2014. Recursive cognition as a prelude to language. In Language and Recursion, eds. Francis Lowenthal and Laurent Lefebvre, 27-36. New York: Springer.

Corballis, Michael. 2017. The Truth About Language. What it is and Where it Came From. Chicago: The University of Chicago Press.

Cosmides, Leda and John Tooby. 2000. Consider the Source: The Evolution of Adaptations for Decoupling and Metarepresentation. In $\mathrm{Me}$ tarepresentations: A Multidisciplinary Perspective, ed. Dan Sperber, 53-115. New York: Oxford.

Dennett, Daniel. 1983. Intentional systems in cognitive ethology: the "Panglossian paradigm" defended. Behavioral and Brain Science 6: 343-390.

Dennett, Daniel. 1996. Kinds of Minds: Toward an Understanding of Consciousness. New York: Basic Books

Dickinson, A. and B. W. Balleine. 2000. Causal cognition and goaldirected action. In The Evolution of Cognition, eds. C. Heyes, L. Huber, 185-204. Cambridge, Mass.: Bradford Books.

Eemeren, F. H. van. 2018. Argumentation Theory: A Pragmadialectical Perspective. Cham: Springer.

Eemeren, F. H. van and Rob Grootendorst. 2004. A Systematic Theory of Argumentation. The Pragma-Dialectical Approach. New York: Cambridge University Press.

Enfield, Nick. 2013. Relationship Thinking. Agency, Enchrony and Human Sociality. New York: Oxford University Press.

Hamblin, Charles. 1970. Fallacies. London: Methuen and Co Ltd.

Henrich, Joseph. 2016. The Secret of Our Success. Princeton: Princeton University Press.

Hoppitt, William and Kevin Laland. 2013. Social Learning. An Introduction to Mechanism, Methods, and Models. Princeton: Princeton University Press.

Godfrey-Smith, Peter. 1996. Complexity and the Function of Mind in Nature. Cambridge: Cambridge University Press.

Pinker, Steven and Ray Jackendoff. 2005. The faculty of language. What's special about it? Cognition 95: 201-236.

Rieke, Richard and Malcolm Sillars. 1993. Argumentation. Critical Decision Making. Third Edition. New York: Harper Collins College Publishers.

Rigotti, Eddo and Sara Greco. 2019. Inference in Argumentation. A Topics-Based Approach to Argument Schemes. Cham: Springer.

Rivano, Emilio. 1999. De la argumentación. Santiago: Bravo y Allende Editores. 
Roeper, Tom and Margaret Speas (eds.). 2015. Recursion: Complexity in Cognition. Cham: Springer.

Searle, John. 2010. Making the Social World. The Structure of Human Civilization. New York: Oxford.

Sterelny, Kim. 2003. Thought in a Hostile World. The Evolution of Human Cognition. Oxford: Blackwell Publishing.

Toulmin, Stephen. 1958. The Uses of Argument. Cambridge: Cambridge University Press.

Toulmin, Stephen, Richard Rieke, and Allen Janik. 1979. An Introduction to Reasoning. New York: Macmillan Publishing Co., Inc.

Walton, Douglas and Thomas Gordon. 2012. The Carneades Model of Argument Invention. Pragmatics \& Cognition 20(1): 1-31.

Walton, Douglas and Erik Krabbe. 1995. Commitment in Dialogue. Basic Concepts of Interpersonal Reasoning. New York: SUNY Press. 